\title{
OPTIMAL PLANNING OF MEDIUM VOLTAGE DISTRIBUTION NETWORKS IN THE PRESENCE OF WIND POWER GENERATIONS
}

\author{
Reza Saberi \\ Birjand University of Technology \\ Iran \\ rsaberi1366@yahoo.com
}

Mehdi Khosravi

Southern Khorasan Electric

Distribution Company (SKEDC)

Iran

khosravi.eng@yahoo.com

\author{
Hamid Falaghi \\ Birjand University of Technology \\ Iran \\ hfalaghi@yahoo.com
}

Taghi Vahidi

Southern Khorasan Electric

Distribution Company (SKEDC)

Iran

taghivahidi@yahoo.com

\author{
Maryam Ramezani \\ Birjand University of Technology \\ Iran \\ ramezani mr@yahoo.com
}

\author{
Mahdi Dadgar \\ Southern Khorasan Electric \\ Distribution Company (SKEDC) \\ Iran \\ mahdidadgar@yahoo.com
}

\begin{abstract}
The aim of this study is to present a method for solving the planning and expansion problems in the distribution systems in order to determine the location and size of new substations and the development of the existing transformers and to find the optimal structure for feeders and selecting the suitable cross-section for feeders in the presence of wind turbines. The objective function of the problem has been mathematically formulated considering uncertainty conditions in the presence of wind turbines and a pseudo-dynamic method for multi-stage design. Also the genetic algorithm has been used as an optimization toolfor solving the problem. In addition, the performance of the above mentioned method on a distribution network has been discussed as well.
\end{abstract}

\section{INTRODUCTION}

Natural growth of loads and also installing new electrical equipment, as the new consumers, is an important issue in planning and expanding distribution systems. Since the Electric Distribution Companies try to increase their benefits and minimize their investment costs; the designers utilize the new design strategies for their networks in order to focus on demand response economically and provide an energy source with high reliability and also maintain competitive in the market.

Finding a radius network with minimum total cost is a very important issue in designing a medium voltage distribution network. In this case, considering some factors such as size and location of substations, optimal route for feeders, crosssection of feeders, the lines status and transformers capacity, reliability restrictions, and appropriate voltage levels in the load points must be taken into account.

Planning and expansion of a distribution system is a complicated process so that complex mathematical methods and lots of numerical analysis are needed for finding an optimal solution. The aforesaid process contains many local optimal solutions as well. In general, for solving the problems relating to the distribution system designs, two following methods are utilized:

1. Static method: This method considers only a design horizon and specifies the location, type, and the capacity of new equipment required for expansion. In other words, all the design requirements for a specific period are determined.

2. Multi-stage (Dynamic) method: In this method, not only the location, type, and the capacity of the equipment are specified for development; but also the most appropriate time for installing new capacities and expanding the existing capacities are determined so that the demand response needs are met optimally. In fact, by this method, the system design is performed in some continuous steps.

Nowadays, the growth trend in DG units such as wind turbines, photovoltaic systems, etc. and also the increasing need in utilizing this kind of units have caused a complexity for analyzing distribution network design in the presence of these units. DG units are one of the new alternatives for distribution network designers. Using these units in distribution networks will decrease the fixed investment costs by postponing the need for new required facilities. These units also play a key role in decreasing current, power, and losses in the system, and also act as an auxiliary source for high load feeders. They improve the voltage profile and increase the life time of the equipment as well. Installing wind turbines as one of the DG sources in the power systems can change the operation conditions and they have important technical and economical advantages too. Thus, applying traditional design methods in the presence of these units is not a modern approach.

Although there are many methods for designing traditional distribution systems based on the evolutionary algorithms [1], [4]; minor studies relating to the DG units are available [2] in which no specific solution for wind turbines has been studied. Since wind has a probability (uncertainty) nature, the output power of wind turbines will have a probability and uncertainty nature too. Hence, probability methods must be considered for the desired problem analysis due to the uncertainty in the system. One of the famous methods for analyzing the uncertainty systems is the Monte-Carlo method. The main idea of Monte-Carlo simulation is to create a series of empirical samples or utilizing random sequence of numbers. In the aforesaid simulation, a provider of random numbers for probability modelling of events is used. When the number of samples is big enough, sample average can be utilized for estimation via mathematical expectation.

In this research, the impact of wind turbines in designing distribution networks in a probability environment has been modelled and then the genetic algorithm has been utilized as 
a tool for optimization and also the pseudo-dynamic method has been used for multi-stage development. Subsequently, the importance of practical application of these methods has been addressed by implementing the aforesaid methods.

\section{MATHEMATICAL FORMULATION}

The objective function of the above problem contains fixed costs relating to investment and installation, and variable costs related to the operation costs. These costs must be minimized considering the problem constraints. The objective function is stated by the following formula where $\mathrm{T}$ is the period of the problem:

$$
F=(I C+O C) \times T
$$

$I C$ is the total investment costs for transformers, feeders, and wind turbines.

$$
I C=\sum_{i \in s s} I C_{i}^{s s}+\sum_{j \in f d} I C_{j}^{f d}+\sum_{k \in w} I C_{k}^{w}
$$

Where $s s, f d$, and $w$ are the existing and new substations, the existing and new feeders, and wind turbines respectively. $O C$ is the total operation cost and it depends on the generation amount of the wind turbines and the amount of power delivered by the substations.

Since the operation cost for wind turbines is negligible, thus the operation cost of substations has only been considered:

$$
O C=\sum_{i \in s s} E C_{i}^{s s} \times P_{i}^{s s}
$$

Where $E C_{i}^{s s}$ is the price of energy in the power market at the $i$ th substation. $\boldsymbol{P}_{i}^{s s}$ is the power delivered by the $i$ th substation.

The problem contains the following constraints:

1. The substations loading capacity must not exceed the maximum amount:

$$
S_{i}^{s s}<S_{i}^{\max }
$$

2. The feeders capacity shall not exceed the maximum withstanding capacity:

$$
S_{j}^{f d} \leq S_{j}^{\max }
$$

3. The capacity of wind turbines must not exceed the maximum capacity of the generation:

$$
S_{k}^{w} \leq S_{k}^{\max }
$$

4. The voltage amount in the load points must be between the minimum and maximum of the allowed operation voltage:

$$
V_{\min }\left\langle V_{\text {load }} \leq V_{\text {max }}\right.
$$

\section{OPTIMIZATION METHOD}

The objective function for the above mentioned problem is a non-linear function that is optimized by an evolutionary method based upon the genetic algorithm. In this method, first the decision variables including the location and size of the new substations, expansion of the existing substations, feeders' structures, and cross-sections of the feeders are determined in the presence of the wind turbines. The first issue that must be defined for solving the problem is the coding type (chromosome's formation) for the desired problem so that each chromosome is a candidate for solving the problem or a solution for decision variables and it contains the two following parts:

1. The first part includes the decision information about the location and size of substations that contains the genes that are equal to the numbers of candidates and existing substations. Each gene shows the required capacity for installing substation at the desired candidate location. The zero number relating to each gene shows that no substation must be installed at the candidate location. As an example, the following chromosome shows that a $30 \mathrm{KVA}$ substation must be installed at the first candidate location or if there's an existing substation there, it must be expanded meanwhile there's no need any new substation in the second candidate location.

Number of existing and candidate substations

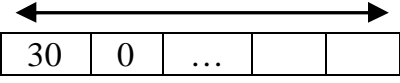

2. The second part shows the status of the network (feeders' structure) and the type of feeder for each section that contains genes that are equal to the existing and new feeders. In fact, each gene relating to the chromosomes of this section shows which feeder must be installed and it can determine the feeder type as well. As another example, the following chromosomes shows that feeder 1 must be installed at the first candidate location and feeder 2 must be installed at the second candidate location.

Number of existing and candidate feeders

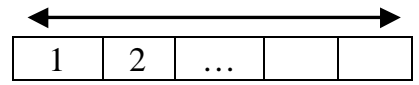

The uncertainty issue must be taken into account in generating electric energy via wind turbines because it assures a proper and fair analysis for the system. In addition, we may have results against the actual behaviour of the system if the system analysis is considered as a real system and analytic simulation is used. Thus, considering uncertainties that enables us to model the actual behaviour of a system is fully obvious.

Monte-Carlo Simulation is one of the popular methods for probability analysis at the power systems and we have 
utilized this method for modelling the uncertainty here. The Monte-Carlo Simulation trend contains the following steps:

1. Determining the inputs and their probability distributions,

2. Sampling the inputs via random numbers and also evaluating the system for each input,

3. Compiling the results and assessing the objective function.

In the problem solving by Monte-Carlo method and due to the uncertainty in the output powers of the wind turbines that is because of the wind nature; the power delivered by the substations and also the operation costs will have probability nature. Here, we have considered a normal distribution with a specified mean and variance for the output power of the wind turbines as the inputs for the Monte-Carlo method meanwhile the normal distribution is utilized as probability variations at the aforesaid method. In the next step, a sampling from the output power of the wind turbines using the production process of uniformed number and operation costs are assessed for each sample. Hence, the desired operation costs will have a probability distribution. Therefore, following formulas can be used for calculating the mean and variance of the operation costs in each step and finally, the mean of operation costs may be used for calculating the objective function:

$$
\begin{gathered}
\operatorname{Var}(O C)=\frac{1}{N-1} \sum_{i=1}^{N}\left(O C_{i}-O C_{a v g}\right)^{2} \\
O C_{\text {avg }}=\frac{1}{N} \sum_{i=1}^{N}\left(O C_{i}\right)
\end{gathered}
$$

Where $N$ is the number of the iterations at the Monte-Carlo Method.

Algorithm is begun by producing a random population and then the objective function is calculated for this population. The genetic algorithm utilizes three rules named elite selection, mutation, and cross-over for creating child for the next generation. Hence, the population is evolved toward an optimal solution.

\section{MULTI-STAGE DEVELOPMENT}

In this paper, the pseudo-dynamic method has been firstly used for multi-stage design so that the period of case study is divided to some intervals. Then the design issue is solved for the whole period using the genetic algorithm and finally all the new equipment that must be installed and also the existing equipment that must be expanded to meet the requirements of load growth are obtained for the whole design period. After that, for the intervals that are located between the aforesaid intervals of the selected equipment (relating to the whole period), genetic algorithm is used for solving the optimization issue. This trend is started from the end of interval and come back to the beginning of the interval.

\section{A numerical example}

The stated design method has been applied to a $20 \mathrm{KV}$ distribution system. This system has a $30 \mathrm{KVA}$ substation that is expandable to $60 \mathrm{KVA}$. It contains two wind turbines with the capacity of 4 MVA as well. The design of the network has been studied for a six-year period and this period is divided to three equal intervals as shown in figure 1 below:

Figure 1 - the design of the network

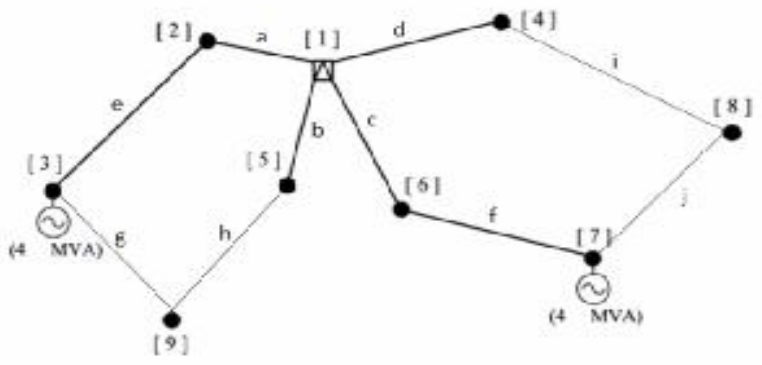

The information for the load points has been presented in Table1:

Table 1 - information of the load points

\begin{tabular}{|c|c|c|c|}
\hline Load point & Stage1 & Stage2 & Stage3 \\
\hline $\mathbf{2}$ & 5.2 & 6.3 & 7.6 \\
\hline $\mathbf{3}$ & 4.1 & 4.4 & 5.1 \\
\hline $\mathbf{4}$ & 5.1 & 5.5 & 6.5 \\
\hline $\mathbf{5}$ & 3.2 & 3.4 & 4.1 \\
\hline $\mathbf{6}$ & 4.6 & 5 & 6.5 \\
\hline $\mathbf{7}$ & - & - & 3.1 \\
\hline $\mathbf{8}$ & - & 2.3 & 3.8 \\
\hline
\end{tabular}

The technical and economical information of the feeders has been presented in Table2:

Table2 - Technical and economical information of the feeders

\begin{tabular}{ccccc}
\hline TYPE & $\mathbf{R}(\mathbf{o h m} / \mathbf{k m})$ & $\mathbf{X}(\mathbf{o h m} / \mathbf{k m})$ & Capacity(MVA) & $\mathbf{C o s t}(\mathbf{M} \mathbf{\$} / \mathbf{k m})$ \\
\hline $\mathbf{1}$ & 0.16 & 0.25 & 12 & 0.1 \\
$\mathbf{2}$ & 0.06 & 0.21 & 18 & 0.15 \\
\hline
\end{tabular}

The design and development of the system is studied for the two following status:

1. Without considering wind turbines: in this case, the feeders $a, b, c, d$, and $e$ must be upgraded and feeders $h$ and $i$ must be installed. In addition, the substation must be upgraded too.

2. By considering wind turbines: In this case, only the feeder $a$ must be upgraded and the feeders $g$ and $j$ must be installed and there's no need to upgrade the substation. 
The network structure in the two aforesaid cases is different meanwhile the need for upgrading the substation in the presence of the wind turbines has been postponed. This advantage is more attractive when expanding the existing substation is not possible for the practical and geographical reasons.

Figure2 exhibits the design cost for the two above cases in the different intervals:

Figure2- design cost for two statuses

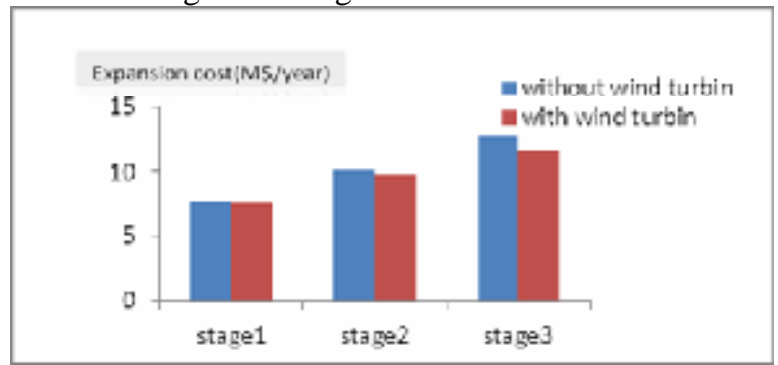

The system expansion costs in the presence of wind turbines are less than the status that there's no wind turbine as shown in the figure.

\section{CONCLUSION}

In this paper, a model for designing distribution system in the presence of wind turbines has been stated so that the genetic algorithm for optimizing objective function and a pseudo-dynamic method for multi-stage expansion have been utilized. As described in the paper, the presence of wind turbines in planning and expansion of the distribution systems can be led to an expansion plan with less costs and more reliability.

\section{REFERENCES}

[1] S. Najafi, S. H. Hosseinian, M. Abedi, A. Vahidnia, and S. Abachezadeh, 2009, "A framework for optimal planning in large distribution networks", IEEE Transactions Power System, vol. 24, no. 2, pp. 10191028.

[2] S. Haffner, L. F.A. Pereira, L. A. Pereira, and L. S. Barreto, 2008, "Multistage model for distribution expansion planning with distributed generation", IEEE Transactions Power Delivery, vol. 23, no. 2, pp.915923.

[3] N. G. Boulaxis and M. P. Papadopoulos, 2002, "Optimal feeder routing in distribution system planning using dynamic programming technique and GIS facilities", IEEE Transactions Power Delivery, vol. 17 , no. 1, pp. 242-247.

[4] W. M. Lin, Y. S. Su, and M. T. Tsay, 1998, "Genetic algorithm for optimal distribution systems planning", IEEE Transactions Power Delivery, vol. 11, no. 4, pp. 224-231. 